\title{
Multibaryons in the Skyrme model ${ }^{*}$
}

\author{
Norberto N. Scoccola \\ Physics Dept., Comisión Nac. Energía Atómica, Libertador 8250, (1429) Bs.As., Argentina \\ and \\ Universidad Favaloro, Solís 453, (1078) Buenos Aires, Argentina.
}

\begin{abstract}
Low-lying multibaryon configurations are studied within the bound state approach to the $S U(3)$ Skyrme model. We use approximate ansätze for the static background fields based on rational maps which have the same symmetries of the exact solutions. To determine the explicit form of the collective Hamiltonians and wave functions we only make use of these symmetries. Thus, the expressions obtained are also valid in the exact case. On the other hand, the meson bindings, inertia parameters and hyperfine splitting constants we calculate do depend on the detailed form of the ansätze and are, therefore, approximate. Using these values we compute the low-lying spectra of multibaryons with $B \leq 9$ and strangeness 0 and $-B$. With these results the stability of some multilambda configurations is discussed.
\end{abstract}

\section{INTRODUCTION}

In the last few years there have been several important developments in the determination of the lowest energy multiskyrmion configurations [1] 3]. This type of solutions are essential for the understanding of multibaryons and, perhaps, nuclei in the framework of the topological chiral soliton models. So far, these models have proven to be useful for the description of quantities such as the masses, strong and electromagnetic properties of the octet and decuplet baryons, baryon-baryon interactions, etc. (see e.g. Refs. [4.5] and references therein). The knowledge of the properties of the multiskyrmion configurations opens the possibility of studying more complex baryonic objects. In fact, several investigations concerning non-strange multiskyrmion systems have been reported in the literature (see, e.g., Refs. [6 10]). Of particular interest are, however, the strange multibaryons. Perhaps the most celebrated example is the $H$ dibaryon predicted in the context of the MIT bag model more than twenty years ago [11. This exotic has been studied in various other models, including the Skyrme model 12 15, but its existence remains controversial both theoretically and experimentally. It has also been speculated that strange matter could be stable $[16]$. This has lead to numerous investigations of the properties of strange matter in bulk and in finite lumps (for a recent review see Ref. [17]). Moreover, with the new heavy ion colliders there is now the possibility of producing strange multibaryons in the laboratory 18. In this situation the study of multibaryon systems within the $S U(3)$ Skyrme model appears to be very interesting. For general soliton configurations this is a quite hard numerical task since one has to deal with several coupled partial differential equations. However, the problem is greatly simplified if one introduces the (approximate) rational maps ansätze [19] for the multiskyrmion configurations. The construction of these ansätze is based on the analogy between BPS monopoles and skyrmions and requires that the approximate solutions have the same symmetries than the exact numerical ones. In fact, it is now known [1] 3] that up to $B=9$ these configurations are very symmetric. Namely, for $B=2$ the solution corresponds to an axially symmetric torus while configurations with $B=3-9$ possess the symmetries of the platonic polyhedra (e.g. tetrahedron for $B=3$, etc). In contrast with the exact solution, however, the rational map approximation assumes that the modulus of the static pionic field is radially symmetric while its direction depends only on the polar coordinates. In this contribution we will report on how to describe multibaryon states in the $S U(3)$ Skyrme model using these approximate ansätze.

\section{SYMMETRIC MULTISKYRMIONS AND RATIONAL MAPS}

A rational map of order $N$ is a map of $S^{2} \rightarrow S^{2}$ of the form

$$
R_{N}(z)=\frac{p(z)}{q(z)}
$$

where $p, q$ are polynomials of degree at most $N$ in the stereographic coordinate $z=\tan (\theta / 2) \exp (i \phi)$. It was shown by Donaldson [20 that there is a one-to-one correspondence between BPS monopoles of order $k$ and rational maps

*Cont. to the Proc. of "Hadron Physics 99". Coimbra, Portugal. September 10- 15, 1999 to be published by AIP. 
of degree $N=k$. Using the analogy between this type of monopoles and the skyrmions, the authors of Ref. 19] proposed the following ansätze for the static soliton chiral field

$$
U_{N}^{r a t .}(\vec{r})=\exp \left[i \vec{\tau} \cdot \hat{n}_{N} F(r)\right]
$$

where

$$
\hat{n}_{N}=\left(\frac{2 \Re\left(R_{N}\right)}{1+\left|R_{N}\right|^{2}}, \frac{2 \Im\left(R_{N}\right)}{1+\left|R_{N}\right|^{2}}, \frac{1-\left|R_{N}\right|^{2}}{1+\left|R_{N}\right|^{2}}\right)
$$

Replacing Eq.(11) in the Skyrme model effective action

$$
\Gamma_{e f f}=\frac{f_{\pi}^{2}}{4} \int d^{4} x \operatorname{Tr} \partial_{\mu} U \partial^{\mu} U^{\dagger}+\frac{1}{32 e^{2}} \int d^{4} x \operatorname{Tr}\left[U^{\dagger} \partial_{\mu} U, U^{\dagger} \partial_{\nu} U\right]^{2}
$$

one gets the following expression for the soliton mass

$$
M_{\text {sol }}=\frac{f_{\pi}^{2}}{2} \int d^{3} r\left[F^{\prime 2}+2 N \frac{\sin ^{2} F}{r^{2}}\left(1+\frac{F^{\prime 2}}{e^{2} f_{\pi}^{2}}\right)+\frac{\mathcal{I}_{N}}{e^{2} f_{\pi}^{2}} \frac{\sin ^{4} F}{r^{2}}\right]
$$

where

$$
\mathcal{I}_{N}=\frac{1}{4 \pi} \int \frac{2 i d z d \bar{z}}{\left(1+|z|^{2}\right)^{2}}\left(\frac{1+|z|^{2}}{1+\left|R_{N}\right|^{2}}\left|\frac{d R_{N}}{d z}\right|\right)^{4}
$$

To obtain the ansatz for a given baryon number $B=N$ one should proceed as follows. First, one constructs the most general map of degree $N$ that has the symmetries of the exact solutions. Then, the resulting $\mathcal{I}_{N}$ has to be minimized with respect to the remaining free parameters. To perform the first step it is useful to recall that under a general $S O(3)$ transformation the stereographic coordinate $z$ transforms as

$$
z \rightarrow \frac{\alpha z+\beta}{-\bar{\beta} z+\alpha}
$$

where $\alpha, \beta$ are entries of the $J=1 / 2$ representation of the corresponding rotation operator. We illustrate the method by considering the case $B=2$. The most general map of degree $N=2$ is

$$
R_{2}=\frac{\mu z^{2}+\nu z+\lambda}{\delta z^{2}+\gamma z+\xi}
$$

If we impose the symmetries of the exact torus configuration (axial symmetry plus $\pi$ rotations around the three cartesian axes) such general form reduces to

$$
R_{2}=\frac{z^{2}-a}{-a z^{2}+1}
$$

The value of $a$ can be now determined by requiring that it should minimize $M_{\text {sol }}$ (that is, $\mathcal{I}_{2}$ ). In this way one finds $a=0$. Thus, the appropriate ansatz is

$$
R_{2}=z^{2}
$$

The explicit expressions of the rational maps corresponding to the other baryon numbers have been given in Ref. [19]. Once such maps are determined, the Euler-Lagrange equation for the soliton profile $F(r)$ can be numerically solved for each baryon number and the multiskyrmion masses $M_{\text {sol }}$ evaluated.

The values of the soliton masses (per baryon number) for the different baryon numbers as calculated using the rational map ansätze are given in Table 1 . For reference, the results corresponding to the skyrmion configurations which fully minimize the static energies [1] 3] and the associate symmetry groups are also given. From this table one observes that the rational map ansätze indeed provide a very good approximation to the exact numerical solutions. 


\section{STRANGE MULTIBARYONS}

We turn now to the study of the strange multibaryons within the $S U(3)$ Skyrme model using the rational map ansätze described in the previous section. For this purpose, the effective action Eq.(3) has to be supplemented with the Wess-Zumino term and some suitable flavor symmetry breaking terms. In the calculations described below we have included terms that account for the different pseudoscalar meson masses and also for the difference between their decay constants.

To extend the model to $S U(3)$ flavor space we use the bound state approach, in which strange baryons appear as bound kaon-soliton systems [21]. Thus, we introduce a generalized Callan-Klebanov ansatz

$$
U=\sqrt{U_{N}} U_{K} \sqrt{U_{N}}
$$

where $U_{N}$ is the $S U(2)$ multiskyrmion field properly embedded into $S U(3)$ and $U_{K}$ is the field that carries the strangeness. Its form is

$$
U_{K}=\exp \left[i \frac{\sqrt{2}}{f_{K}}\left(\begin{array}{cc}
0 & K \\
K^{\dagger} & 0
\end{array}\right)\right]
$$

where $K$ is the usual kaon isodoublet.

In the spirit of the bound state approach we consider first the problem of a kaon field in the background of a static multiskyrmion configuration. To describe such configuration we use the rational map ansatz approximation Eq.(11). Consequently, the ansatz for the kaon field should be

$$
K=k_{N}(r, t) \vec{\tau} \cdot \hat{n} \chi
$$

where $\chi$ is a $1 / 2$ spinor. Replacing Eqs. (10-12) in the effective action and performing the corresponding canonical transformations we obtain a quadratic Hamiltonian whose diagonalization leads to [22]

$$
\left[-\frac{1}{r^{2}} \partial_{r}\left(r^{2} h \partial_{r}\right)+m_{K}^{2}+V-f \epsilon_{N}^{2}-2 \lambda \epsilon_{N}\right] k(r)=0
$$

The radial functions $f, h, \lambda$ and $V$ depend on the baryon number $B$ only through the integral $\mathcal{I}_{N}$. Their explicit expressions can be found in Ref. [22].

Eq.(13) has been solved numerically for different values of $B$ using the values of Ref. [23] for $f_{\pi}$ and $e$ and setting $m_{K}$ and $f_{K} / f_{\pi}$ to their corresponding empirical values. The resulting eigenenergies are listed in Table II. Also listed are the masses (per baryon number) of the corresponding $Y=0$ states in the adiabatic approximation, $M_{Y=0}^{a d i a b} / B=M_{\text {sol }}+\epsilon$. These states are of particular interest since it has been claimed [11,24 that some of them can be stable against strong decays. As a general trend we see that the kaon binding energies $D_{N}^{K}=m_{K}-\epsilon_{N}$ decrease with increasing baryon number. However, as in the case of the energy required to liberate a single $B=1$ skyrmion from the multisoliton background [2]3], we observe some deviation from a smooth behaviour, namely, $D_{4}^{K}>D_{3}^{K}$ and $D_{7}^{K}>D_{6}^{K}$. Consequently, such deviations will be also present in the multiskyrmion mass per baryon. Interestingly, this kind of phenomena has been also observed in some MIT bag model calculations [17. There they are due to shell effects.

Using the values given in Table [I] we obtain

$$
\begin{array}{cl}
M_{2 \Lambda}-2 M_{\Lambda} & =12 \mathrm{MeV} \\
M_{4 \Lambda}-2 M_{2 \Lambda} & =-176 \mathrm{MeV} \\
M_{7 \Lambda}-\left(M_{3 \Lambda}-M_{4 \Lambda}\right) & =-177 \mathrm{MeV}
\end{array}
$$

in the static soliton approximation (i.e. to $\mathcal{O}\left(N_{c}^{0}\right)$ ). These results seem to confirm previous speculations about the stability of the tetralambda in the Skyrme model [24] and opens up the possibility of a stable heptalambda. On the other hand, they indicate that the $H$-particle, although very close to threshold, is not stable.

Within the static multiskyrmion approximation considered so far the spin and isospin quantum numbers of the bound kaon-multiskyrmion systems are not well defined. To recover good spin and isospin quantum numbers we proceed with the standard semi-classical collective quantization [23]. For $B>1$, however, we should introduce independent spin and isospin rotations. The collective Lagrangian reads

$$
L_{\text {coll }}=\frac{1}{2}\left[\Theta_{a b}^{J} \Omega_{a} \Omega_{b}+\Theta_{a b}^{I} \omega_{a} \omega_{b}+2 \Theta_{a b}^{M} \Omega_{a} \omega_{b}\right]-\left(c_{a b}^{J} \Omega_{a}+c_{a b}^{I} \omega_{a}\right) T_{b}
$$

Here, $\vec{\Omega}$ is the angular velocity corresponding to the spin rotation, $\vec{\omega}$ that of the isospin rotation and $T_{b}$ is the kaon spin. $\Theta_{a b}^{J}$ and $\Theta_{a b}^{I}$ are the corresponding moments of inertia while $\Theta_{a b}^{M}$ is an inertia that mixes spin and isospin. 
The constants $c_{a b}^{J}$ and $c_{a b}^{I}$ are the hyperfine splitting constants which for $B=1$ provide the $\Lambda$ - $\Sigma$ mass splitting. The explicit expressions of these inertia and hyperfine splitting tensors in terms of the soliton profile function $F(r)$ and the rational map $R_{N}(z)$ can be found in Ref. [25].

Using the standard definitions for the canonical conjugate momenta

$$
\begin{aligned}
& J_{a}=\frac{\partial L_{c o l l}}{\partial \Omega_{a}}=\Theta_{a b}^{J} \Omega_{b}+\Theta_{a b}^{M} \omega_{b}-c_{a b}^{J} T_{b} \\
& I_{a}=\frac{\partial L_{c o l l}}{\partial \omega_{a}}=\Theta_{a b}^{M} \Omega_{b}+\Theta_{a b}^{I} \omega_{b}-c_{a b}^{I} T_{b}
\end{aligned}
$$

it is rather simply to find the general form of the collective Hamiltonian $H_{\text {coll }}$. Details are given in Ref. [25].

It is important to stress that the structure of the inertia and hyperfine splitting tensors appearing in Eq.(15) is strongly determined by the multiskyrmion symmetries. Using group theory arguments, it can be shown that (for symmetric skyrmions) such tensors are always diagonal. The number of independent diagonal entries, as well as whether the mixing inertias vanish or not, is also fixed by the properties of the corresponding symmetry group $G$. For example, for $B=3$ the three components of the spin and isospin operators transform as the 3-dim irrep $F_{2}$ of the group $T_{d}$. Therefore, there is only one independent component for the spin inertia, one for isospin inertia and one for the mixing inertia. Similar analysis can be done for the hyperfine splittings. For $B=4$, however, $I_{1}, I_{2}$ transform as the 2-dim irrep $E_{g}$ of the group $O_{h}$ while $I_{3}$ as the 1-dim irrep $A_{2 g}$ and the three components of $\vec{J}$ as the 3-dim irrep $T_{1 g}$. Thus, for $B=4$ we should have

$$
\Theta_{11}^{I}=\Theta_{22}^{I} \neq \Theta_{33}^{I} ; \quad \Theta_{11}^{J}=\Theta_{22}^{J}=\Theta_{33}^{J} ; \quad \Theta_{a a}^{M}=0
$$

Finally, we have to determine the collective wave-functions. Their general form must be

$$
\left|J J_{z}, I I_{z}, S\right\rangle=\sum_{J_{3} I_{3} T_{3}} \beta_{J_{3} I_{3} T_{3}}^{J I T} D_{J_{z} J_{3}}^{J} D_{I_{z} I_{3}}^{I} K_{T_{3}}^{T}
$$

where $D_{J_{z} J_{3}}^{J}$ and $D_{I_{z} I_{3}}^{I}$ are $S U(2)$ Wigner functions and $\beta_{J_{3} I_{3} T_{3}}^{J T}$ are some numerical coefficients that have to be fixed by requiring that these wave-functions transform as a 1-dim irrep of $G$. It is very important to notice that such irrep may not coincide with the trivial irrep. As well known when one performs an adiabatic symmetry operation on a skyrmion configuration one can pick a non-trivial phase. These are the so-called Filkenstein-Rubinstein phases. A detailed analysis of these phases for the configurations we are dealing with has been done by Irwin [10]. Using these phases one gets that, except for $B=5,6$, the wavefunctions should transform as the trivial irrep of $G$. For $B=5$ they should transform as the $A_{2}$ irrep of $D_{2 d}$ and for $B=6$ as the $A_{2}$ irrep of $D_{4 d}$.

Having obtained the explicit form of the collective Hamiltonians and wavefunctions, the $\mathcal{O}\left(N_{c}^{-1}\right)$ rotational contribution $E_{r o t}$ to the multiskyrmion masses can be calculated using first order perturbation theory. The numerical values of such contributions to the masses of the lowest lying non-strange baryons are given in Table III while those corresponding to the zero-hypercharge multibaryons are listed in Table IV.

From Table III we note that the quantum numbers of the ground states are consistent with those known for light nuclei with the exception of the odd values $B=5,7,9$. We also observe that the lowest lying state has the lowest possible value of isospin and on the average mass splittings decrease for increasing baryon number. This is a consequence of the fact that, although all the moments of inertia increase with increasing baryon number, the increase of the spin inertia is much faster than that of the isospin one.

Using the values of the rotational corrections to the lowest lying $Y=I=0$ states (some of which are listed in Table IV one can see that stability of the $4 \Lambda$ and the $7 \Lambda$ is not affected by these corrections. For example, for the $4 \Lambda$ there is a decrease of $36 \mathrm{MeV}$ in the binding energy while that of the $7 \Lambda$ is increased by $45 \mathrm{MeV}$.

\section{CONCLUSIONS}

In this contribution we have reported on the description of multibaryons within the bound state approach to the $S U(3)$ Skyrme model. To describe the multiskyrmion backgrounds we have used ansätze based on rational maps. Such configurations are known to provide a good approximation to the exact numerical ones, and lead to a great simplification in the treatment of the kaon-soliton system. An important property of these approximate configurations is that they have the same symmetries as the exact ones. We have shown that the properties of the associated symmetry groups completely determine the explicit form of the collective Hamiltonians (namely, the detailed structure of the inertia and hyperfine splitting tensors). The same happens for the collective wavefunctions. In particular, we have shown how the Filkenstein-Rubinstein phases fix, in a unique way, the one dimensional irreducible representations 
as which each wave function should transform. Thus, the method to obtain the collective Hamiltonians and wave functions described here is also valid in the exact case. On the other hand, the numerical values of the meson bindings and of the independent inertia parameters and hyperfine splitting constants will depend on the detailed form of the ansätze and will be, therefore, approximate.

Using an effective action that provides a good description of the hyperon static properties we have studied the spectra of non-strange and strange multibaryons. In the case of non-strange baryons we found that, for even baryon number, the ground state quantum numbers coincide with those of known stable nuclei. It should be stressed, however, that in our opinion these quite compact multiskyrmion configurations should be interpreted as "multiquark bags" rather than normal nuclei. How these configurations are related with them is not yet clear. Another feature of the predicted spectra is that the low lying non-strange multibaryons always have the lowest possible value of isospin. This can be understood in terms of the behaviour of the inertia tensors as a function of the baryon number. The situation is more complicated in the case of strange particles for which there is a quite delicate interplay between the different terms contributing to the rotational energies. From the calculated spectra of strange multibaryon it results that some $Y=0$ configurations could be stable against strong decays. Such configurations, usually called strangelets, are expected to be seen in RHIC [17,18].

Many of the ideas discussed in the present contribution can be extended to case of heavy flavor (e.g. charmed) multibaryons [26]. In such case, however, a proper treatment requires the use of an effective Lagrangian that accounts for both chiral symmetry and heavy quark symmetry. The present model has been also applied to the study of the binding of the $\eta$ meson to few non-strange baryon systems [27].

We finish with a comment on the Casimir corrections to the multibaryon masses. Although these corrections are not expected to affect in any significant way the kaon eigenvalues and the rotational energies shown here, they might play some role in the determination of the multibaryon binding energies. Within the $S U(2)$ Skyrme model it has been shown 28] that they are responsible for the reduction of the otherwise large $B=1$ soliton mass to a reasonable value when the empirical value of $f_{\pi}$ is used. Here, we have avoided the $B=1$ large mass problem by using the customary method of fitting $f_{\pi}$ to reproduce the nucleon mass [23]. A more consistent approach should certainly use the empirical $f_{\pi}$ and include the Casimir corrections. In this respect, there have been recently some efforts [29] to evaluate the corrections to the $B=1$ mass in the $S U(3)$ Skyrme model. Unfortunately, even in the $S U(2)$ sector, almost nothing is known for $B>1$. This is, of course, a very difficult task since it requires the knowledge of the meson excitation spectrum around the non-trivial multiskyrmion up to rather large energies.

\section{ACKNOWLEDGEMENTS}

The material presented here is based on work done with J.P. Garrahan and M. Schvellinger. Support provided by the grant PICT 03-00000-00133 from ANPCYT, Argentina is acknowledged. The author is fellow of the CONICET, Argentina. He would like to thank the members of the Organizing Committee for their warm hospitality during the workshop.

[1] V.B. Kopeliovich and B.E. Stern, JETP Lett. 45, 203 (1987); J.J.M. Verbaarschot, Phys. Lett. B195, 235 (1987); N.S. Manton, Phys. Lett. B192, 177 (1987).

[2] E. Braaten, S. Townsend and L. Carson, Phys. Lett. B235, 147 (1990).

[3] R.A. Battye and P.M. Sutcliffe, Phys. Rev. Lett. 79, 363 (1997).

[4] I. Zahed and G.E. Brown, Phys. Rep. 142, 1 (1986).

[5] H. Weigel, Int J. Mod. Phys. A11, 2419 (1996).

[6] E. Braaten and L. Carson, Phys. Rev. Lett. 56, 1897 (1986).

[7] L. Carson, Phys. Rev. Lett. 66, 1406 (1991); Nucl. Phys. A535, 479 (1991).

[8] T.S. Walhout, Nucl. Phys. A531, 596 (1991); Nucl. Phys. A547, 423 (1992).

[9] N.R. Walet, Nucl. Phys. A606, 429 (1996).

[10] P. Irwin, hep-th/9804142.

[11] R.L. Jaffe, Phys. Rev. Lett. 38, 195 (1977).

[12] A.P. Balachandran et al, Phys. Rev. Lett. 52, 887 (1984).

[13] V.B. Kopeliovich, B. Schwesinger and B. Stern, Nucl. Phys. A549, 485 (1992).

[14] J. Kunz and P.J. Mulders, Phys. Lett. B215, 449 (1988). 
[15] G.L. Thomas, N.N. Scoccola and A. Wirzba, Nucl. Phys. A575, 623 (1994).

[16] E. Witten, Phys. Rev. D30, 272 (1984).

[17] C. Greiner and J. Schaffner-Bielich, in Heavy Elements and Related New Phenomena, ed. R.K. Gupta and W. Greiner, (World Sci., Singapore); nucl-th/9801062.

[18] K. Borer et al. (NA52 experiment), Phys. Rev. Lett. 72, 1415 (1994); T.A. Armstrong et al. (E864 collaboration), Phys. Rev. Lett. 79, 3612 (1997).

[19] C.J. Houghton, N.S. Manton and P.M. Sutcliffe, Nucl. Phys. B510, 507 (1998).

[20] S.K. Donalson, Com. Math. Phys. 96, 387 (1984).

[21] C. G. Callan and I. Klebanov, Nucl. Phys. B262, 365 (1985); N. N. Scoccola, H. Nadeau, M. Nowak and M. Rho, Phys. Lett. B201, 425 (1988); C. G. Callan, K. Hornbostel and I. Klebanov, Phys. Lett. B202, 269 (1988).

[22] M. Schvellinger and N.N. Scoccola, Phys. Lett. B430, 32 (1998).

[23] G.S. Adkins, C.R. Nappi and E. Witten, Nucl. Phys. B228, 552 (1983).

[24] A.I. Issinskii, B.V. Kopeliovich and B.E. Shtern, Sov. J. Nucl. Phys. 48, 133 (1988).

[25] J.P. Garrahan, M. Schvellinger and N.N. Scoccola, Phys. Rev. D, in print (hep-ph/9906432).

[26] B.V. Kopeliovich and W.J. Zakrzewski, JETP Lett 69, 721 (1999); C.L. Schat and N.N. Scoccola, Phys. Rev. D, in print (hep-ph/9907271); B.V. Kopeliovich and W.J. Zakrzewski, hep-ph/9909365.

[27] D.O. Riska and N.N. Scoccola, Phys. lett. B444, 21 (1998).

[28] B. Moussallam, Ann. Phys. 225, 264 (1993); F. Meier and H. Walliser, Phys.Rep. 289, 383 (1997).

[29] H. Walliser, Phys. Lett. B432, 15 (1998); N.N. Scoccola and H. Walliser, Phys. Rev. D58, 094037 (1998).

TABLE I. Soliton mass per baryon number (in natural units $=6 \pi^{2} f_{\pi} / e$ ) obtained by using the rational map ansatz (APPROX) as compared with the (EXACT) numerical minimization. The corresponding symmetry group $G$ is also listed.

\begin{tabular}{|c|c|c|c|c|}
\hline$B$ & $\mathcal{I}$ & APPROX & EXACT & $G$ \\
\hline 1 & 1 & 1.232 & 1.232 & $O(3)$ \\
\hline 2 & 5.81 & 1.208 & 1.171 & $D_{\infty, h}$ \\
\hline 3 & 13.58 & 1.184 & 1.143 & $T_{d}$ \\
\hline 4 & 20.65 & 1.137 & 1.116 & $O_{h}$ \\
\hline 5 & 35.75 & 1.147 & 1.116 & $D_{2 d}$ \\
\hline 6 & 50.76 & 1.137 & 1.109 & $D_{4 d}$ \\
\hline 7 & 60.87 & 1.107 & 1.099 & $Y_{h}$ \\
\hline 8 & 85.63 & 1.118 & 1.100 & $D_{6 d}$ \\
\hline 9 & 112.83 & 1.123 & 1.099 & $T_{d}$ \\
\hline
\end{tabular}

TABLE II. Kaon eigenenergy $\epsilon_{N}$ and adiabatic approximation to the mass of the zero-hypercharge states (all in $M e V$ ) as a function of the baryon number $B$.

\begin{tabular}{ccc}
\hline \hline$B$ & $\epsilon_{N}$ & $M_{Y=0}^{a d i a b} / B$ \\
\hline 1 & 222 & 1085 \\
2 & 244 & 1091 \\
3 & 255 & 1085 \\
4 & 250 & 1047 \\
5 & 263 & 1067 \\
6 & 267 & 1064 \\
7 & 262 & 1038 \\
8 & 271 & 1055 \\
9 & 276 & 1063 \\
\hline \hline
\end{tabular}


TABLE III. Quantum numbers and rotational energies of the lowest lying $S=0$ states.

\begin{tabular}{|c|c|c|c|}
\hline$B$ & $J^{P}$ & $I$ & $E_{\text {rot }}[\mathrm{MeV}]$ \\
\hline \multirow[t]{2}{*}{3} & $1 / 2^{+}$ & $1 / 2$ & 64 \\
\hline & $5 / 2^{-}$ & $1 / 2$ & 147 \\
\hline \multirow[t]{2}{*}{4} & $0^{+}$ & 0 & 0 \\
\hline & $4^{+}$ & 0 & 173 \\
\hline \multirow[t]{2}{*}{5} & $1 / 2^{+}$ & $1 / 2$ & 28 \\
\hline & $3 / 2^{+}$ & $1 / 2$ & 40 \\
\hline \multirow[t]{2}{*}{6} & $1^{+}$ & 0 & 7 \\
\hline & $3^{+}$ & 0 & 44 \\
\hline \multirow[t]{2}{*}{7} & $7 / 2^{+}$ & $1 / 2$ & 66 \\
\hline & $3 / 2^{+}$ & $3 / 2$ & 98 \\
\hline \multirow[t]{2}{*}{8} & $0^{+}$ & 0 & 0 \\
\hline & $2^{+}$ & 0 & 14 \\
\hline \multirow[t]{2}{*}{9} & $1 / 2^{+}$ & $1 / 2$ & 14 \\
\hline & $5 / 2^{-}$ & $1 / 2$ & 30 \\
\hline
\end{tabular}

TABLE IV. Quantum numbers and rotational energies of the lowest lying $Y=0$ states.

\begin{tabular}{|c|c|c|c|}
\hline$B$ & $J^{P}$ & $I$ & $E_{\text {rot }}[\mathrm{MeV}]$ \\
\hline \multirow[t]{2}{*}{3} & $1 / 2^{+}$ & 1 & 50 \\
\hline & $3 / 2^{-}$ & 0 & 77 \\
\hline \multirow[t]{2}{*}{4} & $0^{+}$ & 2 & 51 \\
\hline & $0^{+}$ & 0 & 72 \\
\hline \multirow[t]{2}{*}{5} & $1 / 2^{+}$ & 1 & 29 \\
\hline & $1 / 2^{-}$ & 1 & 32 \\
\hline \multirow[t]{2}{*}{6} & $0^{+}$ & 2 & 24 \\
\hline & $0^{-}$ & 1 & 26 \\
\hline \multirow[t]{2}{*}{7} & $3 / 2^{+}$ & 2 & 32 \\
\hline & $5 / 2^{+}$ & 1 & 65 \\
\hline \multirow[t]{2}{*}{8} & $0^{+}$ & 2 & 19 \\
\hline & $2^{+}$ & 2 & 31 \\
\hline \multirow[t]{2}{*}{9} & $1 / 2^{-}$ & 2 & 25 \\
\hline & $3 / 2^{-}$ & 2 & 29 \\
\hline
\end{tabular}

